\title{
Life time of resonances in transport simulations
}

\author{
Stefan Leupold \\ Institut für Theoretische Physik, Justus-Liebig-Universität Giessen, \\ D-35392 Giessen, Germany
}

\begin{abstract}
We calculate the life time of a resonance in our recently developed framework for a test-particle description of transport processes for states with continuous mass spectra. The result differs from the expression commonly used in transport simulations but agrees with the one derived by Danielewicz and Pratt relating the life time to the scattering phase shift.
\end{abstract}

PACS numbers: 25.70.Ef, 24.10.Cn, 05.60.-k, 05.70.Ln 


\section{INTRODUCTION}

For the understanding of heavy-ion collisions semi-classical transport theory has become an indispensable tool (cf. e.g. [1],2]). The theoretical foundation of this approach goes back to the pioneering works on non-equilibrium quantum field theory [3 [6]. While former works have focused their attention more or less on the quasi-particle regime (see e.g. [7 13 and references therein) the extension of the formalism to off-shell phenomena has become a topic of growing interest in the last few years 14 22 since it has been realized that the collision rates present in high energetic nucleus-nucleus collisions typically are so large that an onshell approximation seems to be inappropriate. In addition, the resonances excited during the reaction may have large decay widths. Therefore, a representation of these states by stable particles may not be a proper approximation.

The usual approach to solve a transport equation is the representation of the phasespace density by test-particles [1,2] (concerning off-shell extensions see [18 22]). If not only asymptotically stable states but also resonances are simulated by test-particles, one has to attribute a finite life time to those 'resonance test-particles', i.e. one has to demand that they decay after some time of propagation - provided that they had not suffered from a collision prior to their decay. Such resonance test-particles usually have arbitrary invariant masses (chosen according to their spectral distribution). The question which (in general energy- or mass-dependent) life time has to be attributed to the resonance test-particles is under present discussion [23]. The commonly used recipe is to identify the life time with the inverse decay width of the resonance, evaluated for the respective invariant mass. Near threshold the width thus becomes small due to the available phase-space. Hence the life time - if identified with the inverse width - becomes large. In [23] it was suggested to rather calculate the life time from the time delay that the particles suffer which form the resonance. This time delay is given by the energy derivative of the phase shift measured for the particles which scatter by forming the resonance as an intermediate state. This quantity - and therefore also the life time calculated in this way - vanishes near threshold. Hence the two expressions for the life time show a completely different behavior as functions of the invariant mass of the resonance.

Recently a novel approach to the test-particle description of transport processes for states with a continuous mass spectrum has been presented in [21,24]. There the formalism was outlined for a system of non-relativistic (asymptotically stable) states subject to elastic collisions. In principle, however, there is no obstacle to treat also resonances in the same framework and to answer the question about the proper life time for such states. Since in principle the creation and decay of particles is a matter of relativistic field theory we find it more appropriate to present the formalism here for a relativistic system. One of the goals of [21] was the derivation of the (non-relativistic) equations of motion for the test-particles. We do not repeat the straightforward generalization for the relativistic case here. The resulting relativistic equations of motion are given in 20 for energy-momentum independent self-energies and in [22] for arbitrary self-energies. We note that the way in which these equations are obtained in [20,22 is different from our approach [21]. Nonetheless the resulting equations of motion are the same. (This issue is discussed in detail in [21].)

In the next Section we generalize the approach of [21] to a relativistic system (of scalar bosons). In this way we set the stage for a proper treatment of resonances and automatically 
also review the most important points of the formalism outlined in [21]. In Sec. III] we calculate the life time of resonances for various situations. We summarize our results in Sec. IV. An Appendix is added which contains some technical details.

\section{REVIEWING THE DERIVATION OF THE TRANSPORT EQUATION AND THE EFFECTIVE PARTICLE NUMBER DENSITY}

We start out from the Kadanoff-Baym equations [4] for relativistic scalar fields (cf. e.g. [13,20] and references therein)

$$
\begin{aligned}
& \left(-\square_{1}-m^{2}\right) D^{<}\left(1,1^{\prime}\right)=\int d \overline{1}\left[\Sigma^{\mathrm{ret}}(1, \overline{1}) D^{<}\left(\overline{1}, 1^{\prime}\right)+\Sigma^{<}(1, \overline{1}) D^{\mathrm{av}}\left(\overline{1}, 1^{\prime}\right)\right] \\
& \left(-\square_{1}-m^{2}\right) D^{>}\left(1,1^{\prime}\right)=\int d \overline{1}\left[\Sigma^{\mathrm{ret}}(1, \overline{1}) D^{>}\left(\overline{1}, 1^{\prime}\right)+\Sigma^{>}(1, \overline{1}) D^{\mathrm{av}}\left(\overline{1}, 1^{\prime}\right)\right]
\end{aligned}
$$

where we have introduced the two-point functions without ordering

$$
\begin{aligned}
& i D^{<}(x, y)=\langle\phi(y) \phi(x)\rangle, \\
& i D^{>}(x, y)=\langle\phi(x) \phi(y)\rangle
\end{aligned}
$$

and the retarded and advanced quantities

$$
\begin{aligned}
F^{\mathrm{ret}}(x, y) & =\Theta\left(x_{0}-y_{0}\right)\left[F^{>}(x, y)-F^{<}(x, y)\right], \\
F^{\mathrm{av}}(x, y) & =\Theta\left(y_{0}-x_{0}\right)\left[F^{<}(x, y)-F^{>}(x, y)\right]
\end{aligned}
$$

with $F=D, \Sigma$. The self-energy is denoted by $\Sigma$. After Wigner transformation

$$
\bar{F}(X, p)=\int d^{4} u e^{i p u} F(X+u / 2, X-u / 2)
$$

of all quantities a gradient expansion of the Kadanoff-Baym equations is performed. Next one neglects all contributions which are effectively of second or higher order in the derivative with respect to the center-of-mass variable $X$. In this way one ends up with a transport equation (cf. [20] for details of the relativistic case, the corresponding non-relativistic case is discussed in detail in [21])

$$
\sqrt{s} \Gamma \mathcal{A}\left[p^{2}-m^{2}-\operatorname{Re} \bar{\Sigma}^{\mathrm{ret}}, S^{<}\right]-\mathcal{A}\left[\sqrt{s} \Gamma,\left(p^{2}-m^{2}-\operatorname{Re} \bar{\Sigma}^{\mathrm{ret}}\right) S^{<}\right]=\sqrt{s} \Gamma S^{<}-i \bar{\Sigma}^{<} \mathcal{A}
$$

where we have introduced the following real-valued quantities:

two-point functions

$$
S^{<,>}(X, p)=i \bar{D}^{<,>}(X, p)
$$

spectral function円 $\left(s=p^{2}\right)$

\footnotetext{
${ }^{1}$ Note that the definition of the spectral function slightly differs from the non-relativistic version 21]. In the non-relativistic limit there is the following connection between relativistic and nonrelativistic quantities: $\mathcal{A}_{\text {rel }} \rightarrow \mathcal{A}_{\mathrm{nr}} /(4 m), \Gamma_{\text {rel }} \rightarrow \Gamma_{\mathrm{nr}}$.
} 


$$
\mathcal{A}(X, p)=\frac{1}{2}\left(S^{>}(X, p)-S^{<}(X, p)\right)=\frac{\sqrt{s} \Gamma(X, p)}{\left(p^{2}-m^{2}-\operatorname{Re} \bar{\Sigma}^{\operatorname{ret}}(X, p)\right)^{2}+s \Gamma^{2}(X, p)},
$$

width

$$
\Gamma(X, p)=\frac{1}{\sqrt{s}} \frac{i}{2}\left(\bar{\Sigma}^{>}(X, p)-\bar{\Sigma}^{<}(X, p)\right) .
$$

A typical example for the width and the corresponding spectral function is depicted in Figs. 11 and 2, respectively, for a toy model displaying the main features of the $\Delta$ baryon. Width and spectral function start to deviate from zero at threshold (here pion plus nucleon mass). The spectral function grows until the nominal resonance mass is reached. The width grows beyond that point. The large energy behavior is chosen such that $\sqrt{s} \Gamma$ finally reaches a constant value. (Note that the displayed mass range for $\Gamma$ is larger than the one for the spectral function.) Details of the calculation for $\Gamma$ (and of the corresponding real part of the retarded self-energy) are deferred to the Appendix. They form the basis of all the plots which exemplify the relevant quantities.

It is worth noting that the spectral function is normalized

$$
\int_{-\infty}^{+\infty} \frac{d p_{0}}{\pi} p_{0} \mathcal{A}=\int_{0}^{\infty} \frac{d\left(p_{0}^{2}\right)}{\pi} \mathcal{A}=1
$$

if the real part of the retarded self-energy is connected to the width (imaginary part) via a (in general $n$-times subtracted) dispersion relation

$$
\frac{\partial^{n}}{\partial p_{0}^{n}} \operatorname{Re} \bar{\Sigma}^{\mathrm{ret}}\left(X ; p_{0}, \vec{p}\right)=\frac{\partial^{n}}{\partial p_{0}^{n}} \mathcal{P} \int \frac{d k_{0}}{\pi} \frac{1}{p_{0}-k_{0}} \sqrt{k_{0}^{2}-\vec{p}^{2}} \Gamma\left(X ; k_{0}, \vec{p}\right)
$$

where $\mathcal{P}$ denotes the principal value. An example for the real part of the retarded self-energy is shown in Fig. 3 (cf. the Appendix for details).

The generalized Poisson bracket used in (2.8) is defined as

$$
[A, B]=\partial_{X_{0}} A \partial_{p_{0}} B-\partial_{p_{0}} A \partial_{X_{0}} B-\vec{\nabla}_{X} A \vec{\nabla}_{p} B+\vec{\nabla}_{p} A \vec{\nabla}_{X} B
$$

Note that the drift term inherent to any kinetic equation is hidden in

$$
\left[p^{2}-m^{2}, B\right]=-2 p_{0} \partial_{X_{0}} B-2 \vec{p} \cdot \vec{\nabla}_{X} B
$$

The r.h.s. of (2.8) can be expressed via the common gain and loss terms using the definitions (2.10) and (2.11):

$$
\sqrt{s} \Gamma S^{<}-i \bar{\Sigma}<\mathcal{A}=\underbrace{\frac{i}{2} \bar{\Sigma}>S^{<}}_{\text {loss }}-\underbrace{\frac{i}{2} \overline{\Sigma^{<}} S^{>}}_{\text {gain }} .
$$

In the most general case of an interacting multi-particle state away from thermal equilibrium, the transport equation (2.8) is a rather involved integro-differential equation. Within 
the quasi-particle approximation a quite successful method to solve the corresponding transport equation is to use a test-particle ansatz for the phase-space density [1,2]. From the drift and (generalized) Vlasov terms of the transport equation one deduces the equations of motion for the test-particles while the scattering integrals yield the appropriate cross-sections for the collisions among the test-particles. For the case at hand, i.e. beyond the quasi-particle approximation, it is suggestive to use also a test-particle representation

$$
f(t, \vec{x} ; p) \sim \sum_{i} \delta^{(3)}\left(\vec{x}-\vec{x}_{i}(t)\right) \delta\left(p_{0}^{2}-E_{i}^{2}(t)\right) \delta^{(3)}\left(\vec{p}-\vec{p}_{i}(t)\right) .
$$

Note that here in contrast to the quasi-particle approximation the test-particles are allowed to have arbitrary energies not connected to their respective three-momentum by any onshell condition. The remaining question is which quantity $f$ should be represented by testparticles.

So far we have reviewed the derivation of the transport equation (2.8) as outlined in more detail in [20]. Albeit starting from the same transport equation we differ from the approach of [20,22] in what follows concerning the interpretation and use of this equation. In [20,22] the transport equation was merely used as a tool to determine the evolution of test-particles between collisions. In the approach presented here and in 21] we adopt the point of view that the transport equation (2.8) once solved as exactly as possible provides the appropriate propagation, scattering and decays of the test-particles. The differences between [20] and our approach are discussed in more detail in [21]. One difference concerns exactly the question which quantity $f$ should be represented by test-particles.

There is an obvious constraint for such a quantity: the corresponding number of testparticles

$$
N_{t p}=\int d^{3} x \int \frac{d^{4} p}{(2 \pi)^{4}} p_{0} f(t, \vec{x} ; p)
$$

has to remain constant during the propagation of the test-particles and also for elastic scattering events encoded by $\bar{\Sigma}_{\text {elast }}^{<,>}$. Only inelastic scatterings and particle decays both contained in

$$
\bar{\Sigma}_{\text {inelast }}^{<,>}=\bar{\Sigma}^{<,>}-\bar{\Sigma}_{\text {elast }}^{<,>}
$$

should change the number of test-particles $N_{t p}$. For the following considerations we need an important property of the elastic scattering part of the self-energies which is a consequence of detailed balance:

$$
\int \frac{d^{4} p}{(2 \pi)^{4}}\left(i \bar{\Sigma}_{\text {elast }}^{<} S^{>}-i \bar{\Sigma}_{\text {elast }}^{>} S^{<}\right)=0
$$

A natural choice for $f$ seems to be

$$
f(t, \vec{x} ; p) \stackrel{?}{=} S^{<}(t, \vec{x} ; p) .
$$

Indeed, the full Kadanoff-Baym equations (2.1, 2.2) conserve (2.18) with the choice (2.21) as long as only elastic interactions are present. (This was the reason for the choice (2.21) in 
[20,22].) However, we no longer deal with the full Kadanoff-Baym equations but with the transport equation (2.8) obtained from the former by gradient expansion. From (2.8) it is straightforward to get

$$
\begin{aligned}
& \frac{d}{d t} \int d^{3} x \int \frac{d^{4} p}{(2 \pi)^{4}} p_{0} S^{<}(t, \vec{x} ; p) \\
& =\frac{d}{d t} \int d^{3} x \int \frac{d^{4} p}{(2 \pi)^{4}} p_{0} K S^{<}+\int d^{3} x \int \frac{d^{4} p}{(2 \pi)^{4}} \frac{1}{4 \sqrt{s} \Gamma \mathcal{A}}\left(i \bar{\Sigma}^{<} S^{>}-i \bar{\Sigma}^{>} S^{<}\right)
\end{aligned}
$$

with

$$
K=\frac{1}{2 p_{0}}\left(\frac{\partial \operatorname{Re} \bar{\Sigma}^{\mathrm{ret}}}{\partial p_{0}}+\frac{p^{2}-m^{2}-\operatorname{Re} \bar{\Sigma}^{\mathrm{ret}}}{\sqrt{s} \Gamma} \frac{\partial(\sqrt{s} \Gamma)}{\partial p_{0}}\right) .
$$

Even if the self-energies are restricted to their elastic parts, the r.h.s. of (2.22) does not vanish. In contrast, for the choice

$$
f=S_{\text {eff }}^{<}:=2 \sqrt{s} \Gamma \mathcal{A}(1-K) S^{<}
$$

we deduce from (2.8) (cf. [21])

$$
\frac{d}{d t} \int d^{3} x \int \frac{d^{4} p}{(2 \pi)^{4}} p_{0} S_{\text {eff }}^{<}(t, \vec{x} ; p)=\int d^{3} x \int \frac{d^{4} p}{(2 \pi)^{4}} \frac{1}{2}\left(i \bar{\Sigma}^{<} S^{>}-i \bar{\Sigma}^{>} S^{<}\right) .
$$

Using (2.20) we find that this time derivative only deviates from zero in the presence of inelasticities. Hence we conclude that (2.24) is a proper choice for the test-particle number density. Note that the transport equation (2.8) constitutes an effective theory derived from the underlying full quantum field theory by gradient expansion. In this approximation scheme the parts of $S^{<}(X, p)$ with fast oscillations in $X$ are neglected. It is not hard to imagine that such a neglect necessitates the redefinition of conserved quantities as - in our case at hand - the particle number density. In the following we will refer to $S_{\text {eff }}^{<}$as the effective particle number density.

It is fortunate to rewrite the transport equation in terms of this quantity:

$$
\begin{aligned}
& {\left[p^{2}-m^{2}-\operatorname{Re} \bar{\Sigma}^{\mathrm{ret}}, \frac{S_{\mathrm{eff}}^{<}}{1-K}\right]-\frac{1}{\sqrt{s} \Gamma}\left[\sqrt{s} \Gamma,\left(p^{2}-m^{2}-\operatorname{Re} \bar{\Sigma}^{\mathrm{ret}}\right) \frac{S_{\mathrm{eff}}^{<K}}{1-K}\right]} \\
& =\frac{i \bar{\Sigma}>}{2 \sqrt{s} \Gamma \mathcal{A}(1-K)} S_{\mathrm{eff}}^{<}-i \bar{\Sigma}^{<} S^{>}
\end{aligned}
$$

with

$$
S^{>}=2 \mathcal{A} b
$$

and the Bose enhancement factor

$$
b=1+\frac{S_{\text {eff }}^{<}}{4 \sqrt{s} \Gamma \mathcal{A}^{2}(1-K)} .
$$


To obtain (2.27) we have expressed $S^{>}$in terms of $\mathcal{A}$ and $S^{<}$according to (2.10). $S^{<}$ is expressed in terms of $S_{\text {eff }}^{<}$using (2.24). Note that in (global) thermal equilibrium the following relations hold:

$$
\left.\begin{array}{rl}
S^{<}(p) & =2 n_{B}\left(p_{0}\right) \mathcal{A}(p) \\
S_{\text {eff }}^{<}(p) & =2 n_{B}\left(p_{0}\right) 2 \sqrt{s} \Gamma(p) \mathcal{A}^{2}(p)(1-K(p)) \\
i \bar{\Sigma}^{<}(p) & =2 n_{B}\left(p_{0}\right) \sqrt{s} \Gamma(p) \\
b(p) & =1+n_{B}\left(p_{0}\right)
\end{array}\right\} \text { thermal equilibrium }
$$

where we have introduced the Bose distribution $n_{B}$.

The transport equation (2.26) forms the basis of the following considerations.

\section{LIFE TIME OF RESONANCES}

\section{A. Vacuum case}

For the most general off-equilibrium situation the transport equation (2.26) is rather complicated. To focus on the aspect relevant for our purpose we restrict ourselves to the following scenario: A bunch of resonances (which do not interact with each other) with invariant mass $\sqrt{s}=\sqrt{p^{2}}$ is uniformly distributed in vacuum and their decay time is determined. This allows for drastic simplifications of the transport equation. First of all, any dependence on $\vec{x}$ vanishes. Second, the gain rate $i \bar{\Sigma}^{<}$vanishes since no new resonances are formed. Finally, the self-energies do not depend on time. Thus we get

$$
-\left(2 p_{0}-\frac{\partial \operatorname{Re} \bar{\Sigma}^{\mathrm{ret}}}{\partial p_{0}}\right) \frac{1}{1-K} \partial_{t} S_{\mathrm{eff}}^{<}+\frac{\partial(\sqrt{s} \Gamma)}{\partial p_{0}} \frac{p^{2}-m^{2}-\operatorname{Re} \bar{\Sigma}^{\mathrm{ret}}}{\sqrt{s} \Gamma(1-K)} \partial_{t} S_{\mathrm{eff}}^{<}=\frac{i \bar{\Sigma}^{>}}{2 \sqrt{s} \Gamma \mathcal{A}(1-K)} S_{\text {eff }}^{<} .
$$

Using (2.11) (note: $\left.i \bar{\Sigma}^{<}=0\right)$ and (2.23) one ends up with the very simple relation

$$
-2 p_{0} \partial_{t} S_{\mathrm{eff}}^{<}=\frac{i \bar{\Sigma}>}{2 \sqrt{s} \Gamma \mathcal{A}(1-K)} S_{\mathrm{eff}}^{<}=\frac{1}{\mathcal{A}(1-K)} S_{\mathrm{eff}}^{<}
$$

from which the life time can be immediately read off:

$$
\tau=2 p_{0} \mathcal{A}(1-K) .
$$

As an example the life time of the $\Delta$ baryon is given in Fig. 4 (full line). The expression on the r.h.s. can be rewritten by introducing the phase shift $\delta$ via

$$
\tan \delta=\frac{-\sqrt{s} \Gamma}{p^{2}-m^{2}-\operatorname{Re} \bar{\Sigma}^{\mathrm{ret}}} .
$$

One finds 


$$
\tau=\frac{\partial \delta}{\partial p_{0}} .
$$

$\delta$ is displayed in Fig. 5. It shows the typical behavior of a resonant scattering phase shift (here of pion-nucleon scattering), i.e. it is small for low invariant masses and rises strongly in the vicinity of the resonance peak mass. There it becomes $90^{\circ}$.

Relation (3.5) agrees with the one found by Danielewicz and Pratt [23] but is in striking disagreement with the one commonly used in simulations of nucleus-nucleus collisions (see also the discussion in [26]), namely

$$
\tau \stackrel{?}{=} \frac{p_{0}}{\sqrt{s} \Gamma}
$$

(Note that in the rest frame of the resonance the latter expression reduces to $1 / \Gamma$ while the r.h.s. of (3.3) becomes $2 \sqrt{s} \mathcal{A}(1-K)$.)

Relation (3.5) can be easily understood if one considers the scattering of a wave packet on a potential [25]: At large distances $r$ from the interaction region the scattered wave is given by

$$
\int d^{3} k \psi(\vec{k}-\vec{p}) \frac{e^{i k r}}{r} f\left(E_{k}, \theta_{k}\right) e^{-i E_{k} t}
$$

where $\psi$ denotes the wave packet amplitude. For an amplitude sharply peaked near $\vec{k}=\vec{p}$ one might expand the scattering amplitude $f$ around $E_{p}:{ }^{2}$

$$
\begin{aligned}
f\left(E_{k}\right) & =\exp \left[\ln f\left(E_{k}\right)\right] \approx \exp \left[\ln f\left(E_{p}\right)+\left(E_{k}-E_{p}\right) \frac{\partial}{\partial E_{p}} \ln f\left(E_{p}\right)\right] \\
& =f\left(E_{p}\right) \exp \left[\left(E_{k}-E_{p}\right) \frac{\partial}{\partial E_{p}} \ln f\left(E_{p}\right)\right] .
\end{aligned}
$$

Hence the time delay which the scattered wave packet suffers is given by

$$
\operatorname{Im}\left[\frac{\partial}{\partial E_{p}} \ln f\left(E_{p}\right)\right]=\frac{\partial \delta}{\partial E_{p}}
$$

where we have introduced the phase shift (for a single channel with angular momentum $l$ ) via

$$
f=\frac{1}{p} \sin \delta P_{l}\left(\cos \theta_{p}\right) e^{i \delta}
$$

Suppose now that two particles scatter by forming a resonance. In this case the time delay caused by their scattering can be identified with the life time of the formed resonance. This is exactly the essence of relation (3.5).

\footnotetext{
${ }^{2}$ For simplicity we suppress the angular dependence.
} 


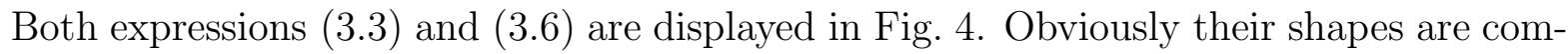
pletely different from each other. The most striking difference is their respective threshold behavior. While $\tau$ as given in (3.3) vanishes at threshold, in contrast $1 / \Gamma$ diverges there as the width vanishes. In addition, $\tau$ is peaked in the vicinity of the nominal resonance mass. There $\tau$ is roughly twice as large as $1 / \Gamma$. The latter quantity decreases monotonically in that region. In spite of their completely different shapes, it is interesting to realize that there are intuitive interpretations for both expressions (3.3) and (3.6). If a resonance with arbitrary invariant mass is considered as a "real particle", it is clear that its life time increases near threshold due to the limited phase-space available for the decay products. This consideration suggests formula (3.6) corresponding to the dashed line in Fig. 6 . If, however, the resonance is considered as a quantum mechanical transient state the uncertainty principle applies. Hence the more the invariant mass of the resonance deviates from its pole mass the less time it is allowed to live. Qualitatively this is the essence of (3.3) displayed by the

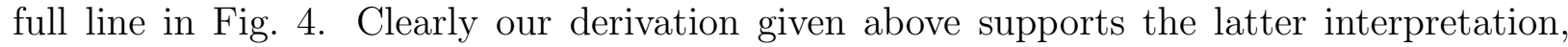
in spite of the fact that what we are aiming at is the appropriate life time of (resonance) test-particles which intuitively are regarded to be closer to real particles than to quantum mechanical states. Nonetheless the transport equation (2.26) derived from the underlying quantum field theory clearly demands that the appropriate life time is given by (3.3) instead of (3.6).

At this stage some words of clarification are necessary. For a sharp resonance it is wellknown (e.g. [27]) that its "life time" is given by $1 / \Gamma_{\text {on-shell }}$ (in its rest frame) which seems to be in line with (3.6) but in contrast to (3.3) which roughly yields twice as much (cf. Fig. (4). This reasoning, however, is misleading. In the preceding paragraphs we studied the life time $\tau$ of a resonance with arbitrary invariant mass. In contrast, the well-known "life time" $\langle\tau\rangle$ of a sharp resonance is a quantity averaged over the allowed energy (or invariant mass) range. As we shall see now both expressions (3.3) and (3.6), albeit completely different in shape, yield the same average value, if the width is sufficiently small. The natural choice for the probability (density) to find a resonance with energy $p_{0}$ is given by the spectral function (2.10). Recalling the normalization (2.12) we define

$$
\langle\tau\rangle:=\int_{0}^{\infty} \frac{d\left(p_{0}^{2}\right)}{\pi} \mathcal{A} \tau .
$$

To evaluate this average for both expressions (3.3) and (3.6) we restrict ourselves to the case of small width, i.e. a sharply peaked resonance. In this case we can neglect any energy dependence of the width and simply evaluate it at the pole mass:

$$
\Gamma_{\text {on-shell }}=\Gamma\left(p_{0}=E_{p}, \vec{p}\right)
$$

with $E_{p}:=\sqrt{m^{2}+\vec{p}^{2}}$. For simplicity we also neglect the real part of the retarded self-energy. As already pointed out in [21] the following relations hold (translated here to a relativistic system):

$$
\left.\begin{array}{c}
\mathcal{A} \\
2 \sqrt{s} \Gamma \mathcal{A}^{2}
\end{array}\right\} \rightarrow \pi \delta\left(p^{2}-m^{2}\right) \operatorname{sgn}\left(p_{0}\right) \quad \text { for } \quad \Gamma, \operatorname{Re} \bar{\Sigma}^{\text {ret }} \rightarrow 0
$$


Hence we find for the expression (3.3)

$$
\langle\tau\rangle=\int_{0}^{\infty} \frac{d\left(p_{0}^{2}\right)}{\pi} \underbrace{2 \sqrt{s} \Gamma \mathcal{A}^{2}(1-K)}_{\rightarrow \pi \delta\left(p^{2}-m^{2}\right)} \frac{p_{0}}{\sqrt{s} \Gamma} \rightarrow \frac{E_{p}}{m \Gamma_{\text {on-shell }}} .
$$

We get the same result using (3.6)

$$
\left\langle\frac{p_{0}}{\sqrt{s} \Gamma}\right\rangle=\int_{0}^{\infty} \frac{d\left(p_{0}^{2}\right)}{\pi} \mathcal{A} \frac{p_{0}}{\sqrt{s} \Gamma} \rightarrow \frac{E_{p}}{m \Gamma_{\text {on-shell }}} .
$$

In the rest frame of the resonance this reduces to the well-known expression?

$$
\langle\tau\rangle=1 / \Gamma_{\text {on-shell }}
$$

Note, however, that this relation provides only an average value of the life time. It does not answer the question how long a resonance with arbitrary invariant mass lives.

Concerning practical applications, i.e. transport simulations with test-particles, the previous considerations suggest the following two possibilities for states with a small width:

1. One might represent $S_{\text {eff }}^{<}$by test-particles having arbitrary invariant masses. For small width the likelihood to produce a test-particle with a mass far away from the pole mass is of course negligibly small. Therefore basically all test-particles will have similar (but not exactly the same) invariant masses. The life time of a test-particle is given by (3.3).

2. The second possibility is to attribute the on-shell mass to all test-particles. In this case the test-particles represent the integrated quantity $\int d\left(p_{0}^{2}\right) S_{\text {eff }}^{<}$instead of $S_{\text {eff }}^{<}$. Consequently all test-particles have to decay with the same integrated (averaged) life time $\langle\tau\rangle=1 / \Gamma_{\text {on-shell }}$.

Obviously, concerning states with a large width only the first possibility is reasonable. From a practical point of view a word of caution is necessary. To realize the first possibility one has to make sure that one has chosen enough test-particles to "fill out" the whole spectral distribution. Especially, if the test-particles in the tails of the spectral distribution are missing one might obtain an average life time which is too high since (cf. Fig. ஐ)

$$
\tau_{s \approx m^{2}} \approx 2 / \Gamma_{\text {on-shell }} \approx 2\langle\tau\rangle .
$$

Hence for states with a small width the second possibility described above seems to be more practical.

It is worth to discuss the role of the factor $(1-K)$ in the expression for the life time (3.3). Due to the contribution (cf. (2.23))

\footnotetext{
${ }^{3}$ It is interesting to mention that even for the exemplifying case of the $\Delta$ baryon displayed in the figures with its quite sizable on-shell width of $120 \mathrm{MeV}$ the average values in (3.14) and (3.15) do not deviate much from each other: $\langle\tau\rangle \approx 1.68 \mathrm{fm} / \mathrm{c},\langle 1 / \Gamma\rangle \approx 1.55 \mathrm{fm} / \mathrm{c}, 1 / \Gamma_{\text {on-shell }} \approx 1.64 \mathrm{fm} / \mathrm{c}$.
} 


$$
\frac{1}{\sqrt{s} \Gamma} \frac{\partial(\sqrt{s} \Gamma)}{\partial p_{0}}
$$

$K$ diverges at threshold, i.e. where $\Gamma$ vanishes. This is shown in Fig. 6. We also deduce from that figure that $(1-K)$ remains positive for all values of $s$. This is a necessary consistency condition since the life time and also the effective particle number density (2.24) should not become negative (cf. the corresponding discussion in [21]). In Fig. 7 we compare the life time $\tau$ from (3.3) with the simpler quantity $2 \sqrt{s} \mathcal{A}$. As can be seen the main effect of $(1-K)$ on $\tau$ is to change the threshold behavior of the spectral function for the $\Delta$ (cf. (A1, A2) )

$$
\mathcal{A} \sim \Gamma \sim k_{\text {rel }}^{3} \sim\left(\sqrt{s}-\left(m_{N}+m_{\pi}\right)\right)^{3 / 2} \quad \text { for } \quad \sqrt{s} \approx m_{N}+m_{\pi}
$$

to

$$
\tau \sim \mathcal{A}(1-K) \sim\left(\sqrt{s}-\left(m_{N}+m_{\pi}\right)\right)^{1 / 2} \quad \text { for } \quad \sqrt{s} \approx m_{N}+m_{\pi} .
$$

This explains the kink of $\tau$ at threshold as compared to the smooth rising of the spectral function. In total, however, $\tau$ and $2 \sqrt{s} \mathcal{A}$ look rather similar, especially if compared to the completely different shape of $1 / \Gamma$ displayed in Fig. 4 .

\section{B. More than one decay channel}

We now turn to an extension of the formalism applicable when there exists more than one decay channel for the resonance. While the total life time is given by (3.3) it is also important to know the partial decay rates (inverse life times) for the various channels. Formally this can be accounted for by realizing that $\bar{\Sigma}^{>}=: \bar{\Sigma}_{\text {tot }}^{>}$is given by a sum of the self-energies corresponding to the different decay channels:

$$
i \bar{\Sigma}_{\text {tot }}^{>}=\sum_{j} i \bar{\Sigma}_{j}^{>}=\sum_{j} 2 \sqrt{s} \Gamma_{j} .
$$

The latter equality makes sense if there are no production channels, i.e. $\bar{\Sigma}_{j}^{<}=0$. Now it is straightforward to deduce from (3.2) the evolution equation

$$
\partial_{t} S_{\mathrm{eff}}^{<}=-\sum_{j} \frac{1}{\tau_{j}} S_{\mathrm{eff}}^{<}
$$

with the partial life times

$$
\tau_{j}=\frac{2 p_{0} \Gamma_{\text {tot }} \mathcal{A}(1-K)}{\Gamma_{j}}=\frac{\Gamma_{\text {tot }}}{\Gamma_{j}} \tau_{\text {tot }}
$$

where we have attributed the index "tot" to all expressions calculated earlier to distinguish them from the expressions belonging to a single one of the decay channels. We note in passing that the probability for the resonance to decay into channel $j$ (branching ratio) agrees with the probability that one would deduce from (3.6):

$$
\frac{\tau_{\mathrm{tot}}}{\tau_{j}}=\frac{\Gamma_{j}}{\Gamma_{\mathrm{tot}}}=\frac{\frac{1}{\Gamma_{\mathrm{tot}}}}{\frac{1}{\Gamma_{j}}} .
$$




\section{In-medium case}

So far, we have restricted our considerations to resonance decays in vacuum neglecting the production channels encoded in $\bar{\Sigma}^{<}$. If we want to study creation and decay of resonances in a medium, we have to take both loss and gain terms, i.e. the full r.h.s. of (2.26) into account. For simplicity we ignore the in general rather lengthy l.h.s. of (2.26) which describes the propagation of the test-particles according to drift term, Vlasov term, etc. For our purposes it is enough to study the simplified evolution equation

$$
-2 p_{0} \partial_{t} S_{\text {eff }}^{<}=\frac{i \bar{\Sigma}>}{2 \sqrt{s} \Gamma \mathcal{A}(1-K)} S_{\text {eff }}^{<}-i \bar{\Sigma}^{<} S^{>} .
$$

In general, $i \overline{\Sigma^{>}}$and $i \bar{\Sigma}^{<}$are connected by detailed balance. For the case of thermal equilibrium this leads to the Kubo-Martin-Schwinger (KMS) boundary condition (cf. e.g. [13])

$$
i \bar{\Sigma}^{<}(p)=i \bar{\Sigma}^{>}(p) e^{-p_{0} / T} .
$$

To simplify the discussion and to get a closer relation to the life time introduced above we assume in the following

$$
i \bar{\Sigma}^{<}(X, p) \ll i \bar{\Sigma}^{>}(X, p)
$$

(concerning the thermal state we turn from a Bose to a Boltzmann distribution). Using (2.11) we find

$$
i \bar{\Sigma}^{>}(X, p) \approx 2 \sqrt{s} \Gamma(X, p) .
$$

The KMS-condition (detailed balance) becomes

$$
i \bar{\Sigma}^{<}(p) \approx 2 \sqrt{s} \Gamma(p) e^{-p_{0} / T}
$$

while (3.25) simplifies to

$$
\partial_{t} S_{\text {eff }}^{<}=-\frac{1}{\tau} S_{\text {eff }}^{<}+\frac{i \bar{\Sigma}^{<}}{p_{0}} \mathcal{A}
$$

where we have replaced $b$ as defined in (2.28) by 1 . Hence we deduce that the effective loss rate is given by $1 / \tau$ while the gain rate is $i \bar{\Sigma}^{<} / p_{0}$. However, since $\sqrt{s} \Gamma(p) / p_{0}$ and not $1 / \tau$ is connected to $i \bar{\Sigma}^{<} / p_{0}$ by detailed balance (3.29) one might worry about the correct thermal limit for the particle number density. Thermal equilibrium, i.e. a stationary solution for $S_{\text {eff }}^{<}$ is reached if gain and loss terms cancel each other:

$$
-\frac{1}{\tau} S_{\mathrm{eff}}^{<}+\frac{i \bar{\Sigma}^{<}}{p_{0}} \mathcal{A} \rightarrow 0 .
$$

Using the definition of the life time (3.3) and the (simplified) KMS-condition (3.29) we end up with

$$
S_{\mathrm{eff}}^{<} \rightarrow 4 \sqrt{s} \Gamma \mathcal{A}^{2}(1-K) e^{-p_{0} / T} \approx 4 \sqrt{s} \Gamma \mathcal{A}^{2}(1-K) n_{B}
$$


where we have replaced the Boltzmann by the Bose function in the last step. Indeed this is the correct thermal limit for $S_{\text {eff }}^{<}$as already presented in (2.29). Hence we have shown that the combination of loss and gain terms on the r.h.s. of the transport equation (2.26) indeed yields the correct thermal limit for the effective particle number density $S_{\text {eff }}^{<}$. Turning the argument around we conclude that attributing the life time (3.3) to a resonance only leads to thermodynamically consistent results if one realizes that the appropriate (effective) particle number density is given by $S_{\text {eff }}^{<}$introduced in (2.24) and not by $S^{<}$. Assuming erroneously that the test-particles which decay according to (3.3) represent $S^{<}$one would essentially solve an evolution equation like

$$
\partial_{t} S^{<}=-\frac{1}{\tau} S^{<}+\frac{i \bar{\Sigma}^{<}}{p_{0}} \mathcal{A} \quad \text { (wrong!) }
$$

instead of (3.30). The thermal limit of the (wrong) equation (3.33) is of course given by

$$
S^{<} \rightarrow 4 \sqrt{s} \Gamma \mathcal{A}^{2}(1-K) e^{-p_{0} / T} \quad \text { (wrong!) }
$$

in contrast to the correct behavior (cf. (2.29))

$$
S^{<} \rightarrow 2 \mathcal{A} n_{B} \approx 2 \mathcal{A} e^{-p_{0} / T}
$$

To conclude we have deduced the expression (3.3) for the life time from the transport equation (2.26) for $S_{\text {eff }}^{<}$. In turn we have also shown that starting from the life time (3.3) for test-particles one has to realize that these test-particles represent $S_{\text {eff }}^{<}$and not $S^{<}$. f

\section{SUMMARY}

After a short review of the derivation of a transport equation from the underlying quantum field theoretical Kadanoff-Baym equations we have focused on the test-particle representation of the transport process. At present this seems to be the most promising tool to solve the transport equation in practice. We have pointed out (repeating the arguments of [21]) that the proper quantity which should be simulated by test-particles, i.e. which counts the test-particles per four-momentum and space volume, is given by the effective particle number density $S_{\text {eff }}^{<}$introduced in (2.24). Once this identification has been made it was straightforward to derive from the transport equation (2.26) the life time of the resonance test-particles. For the vacuum case we obtained expression (3.3) which can be rewritten as (3.5) confirming the result of Danielewicz and Pratt [23]. As can be seen from Fig. 4

\footnotetext{
${ }^{4}$ Note that an exact treatment of the r.h.s. of (3.25) together with the exact KMS-condition (3.26) would have led immediately to the last expression of (3.32). We made the approximations only to get closer contact to the expression for the life time.

${ }^{5}$ Concerning these detailed balance considerations the use of $1 / \Gamma$ for the life time would be compatible with $S^{<}$.
} 
this result for the life time strongly differs from the inverse width prescription commonly used in transport simulations. If more than one decay channel for the resonance has to be considered, the partial life time is given by (3.23). The latter formula also applies if (in a medium) collisional broadening modifies the total width. Note that for the calculation of the life times the required spectral function as well as total and partial widths should be determined self-consistently for the in-medium case. Finally we have shown that the identification of the proper effective particle number density (2.24) and the relation for the life time (3.3) are intimately connected. On the one hand, the life time formula (3.3) is derived from the transport equation (2.26) for the effective particle number density (2.24). On the other hand, starting out from the formula for the life time and assuming thermal equilibrium leads to an expression for the particle number density which agrees with the thermodynamic limit of $S_{\text {eff }}^{<}$.

In [21] it has been shown that the scattering cross sections for off-shell test-particles 9 have to be modified to account for the fact that these test-particles represent the effective particle number density $S_{\text {eff }}^{<}$and not $S^{<}$. There are rather simple rules for the required modification:

1. For each incoming off-shell test-particle the cross section has to be divided by

$$
r=2 \sqrt{s} \Gamma \mathcal{A}(1-K)
$$

where all quantities refer to the (in-medium) properties of the off-shell test-particle, i.e. $\Gamma$ is its total (in-medium) width etc.

2. For each outgoing off-shell test-particle the Pauli blocking/Bose enhancement factor is given by (cf. (2.28)

$$
1 \mp \frac{S_{\text {eff }}^{<}}{4 \sqrt{s} \Gamma \mathcal{A}^{2}(1-K)} .
$$

In the same way the result for the life time can be understood: In complete analogy to the cross section the decay width $\Gamma_{j}$ has to be divided by (4.1). In that way the inverse of that quantity changes from $1 / \Gamma_{j}$ to (3.23) which is our expression for the life time. Whether these new rules for the calculation of cross sections and decay probabilities lead to observable effects in nucleus-nucleus collisions remains to be seen.

\footnotetext{
${ }^{6}$ Here "off-shell test-particles" denote test-particles with arbitrary invariant mass as they appear in the definition of $f$ in (2.17). By chance such test-particles even might be on their mass-shell — still we would call them "off-shell test-particles". This phrase merely denotes a concept than a single particle, namely the concept that "off-shell test-particles" fill the generalized phase-space of four-momentum and coordinate space (weighted by their spectral distribution). In contrast "onshell test-particles" live in usual phase-space. While "off-shell test-particles" represent $f$ "on-shell test-particles" represent $\int d\left(p_{0}^{2}\right) f$. These concepts are also discussed at page 10 (items 1. and 2.).
} 


\section{ACKNOWLEDGMENTS}

I acknowledge fruitful discussions with J. Aichelin, V. Koch, and U. Mosel during the "Hirschegg 2000" workshop. These discussions initiated the work presented here. I also thank W. Cassing, C. Greiner, and S. Juchem for their helpful comments on the manuscript.

\section{APPENDIX A: A SIMPLE MODEL FOR THE $\triangle$ RESONANCE}

To exemplify the formulae given above we study a toy model for the $\Delta$ resonance where spin (and also isospin) is neglected for simplicity. The width is determined by the $\Delta \rightarrow N \pi$ decay channel:

$$
\Gamma(s)=\Gamma_{0} \frac{\beta(s)}{\beta\left(m_{\Delta}^{2}\right)} \quad \text { for } \sqrt{s} \geq m_{N}+m_{\pi}
$$

with

$$
\beta(s)=\frac{k_{\mathrm{rel}}}{s} \frac{\left(k_{\mathrm{rel}} R\right)^{2}}{1+\left(k_{\mathrm{rel}} R\right)^{2}} .
$$

Here the momentum $k_{\text {rel }}$ of $\pi$ and $N$ in the rest frame of the decaying $\Delta$ resonance with invariant mass $\sqrt{s}$ is given by

$$
k_{\mathrm{rel}}=\frac{\left[\left(s-\left(m_{N}+m_{\pi}\right)^{2}\right)\left(s-\left(m_{N}-m_{\pi}\right)^{2}\right)\right]^{1 / 2}}{2 \sqrt{s}} .
$$

In (A2) we have included a Blatt-Weisskopf function [28 parametrized by the length parameter $R$. We adopt the following values to simulate the vacuum decay properties of the $\Delta$ resonance: $m_{N}=940 \mathrm{MeV}, m_{\pi}=140 \mathrm{MeV}, m_{\Delta}=1232 \mathrm{MeV}, \Gamma_{0}=120 \mathrm{MeV}$, and $R=1 \mathrm{fm}$.

Since $\sqrt{s} \Gamma$ becomes constant for large energies the real part of the retarded self-energy can be calculated by a one-time subtracted dispersion relation according to (2.13). We choose the subtraction constant such that the real part of the self-energy vanishes for $s=m_{\Delta}^{2}$. In our simple vacuum model the energy integration in (2.13) can be rewritten in terms of the invariant mass squared. One finally gets:

$$
\operatorname{Re} \bar{\Sigma}^{\mathrm{ret}}(s)=-\left(s-m_{\Delta}^{2}\right) \mathcal{P} \int_{\left(m_{N}+m_{\pi}\right)^{2}}^{\infty} \frac{d s^{\prime}}{\pi} \frac{\sqrt{s^{\prime}} \Gamma\left(s^{\prime}\right)}{\left(s-s^{\prime}\right)\left(m_{\Delta}^{2}-s^{\prime}\right)}
$$




\section{REFERENCES}

[1] G.F. Bertsch and S. Das Gupta, Phys. Rep. 160, 189 (1988).

[2] W. Cassing, V. Metag, U. Mosel, and K. Niita, Phys. Rep. 188, 363 (1990).

[3] J. Schwinger, J. Math. Phys. 2, 407 (1961).

[4] L.P. Kadanoff and G. Baym, Quantum Statistical Mechanics, Benjamin, New York (1962).

[5] P.M. Bakshi and K.T. Mahanthappa, J. Math. Phys. 4, 1, 12 (1963).

[6] L.V. Keldysh, Zh. Eks. Teor. Fiz. 47, 1515 (1964); Sov. Phys. JETP 20, 1018 (1965).

[7] P. Danielewicz, Ann. Phys. 152, 305 (1984).

[8] K. Chou, Z. Su, B. Hao, and L. Yu, Phys. Rep. 118, 1 (1985).

[9] W. Botermans and R. Malfliet, Phys. Rep. 198, 115 (1990).

[10] S. Mrówczyński and P. Danielewicz, Nucl. Phys. B342, 345 (1990).

[11] S. Mrówczyński and U. Heinz, Ann. Phys. 229, 1 (1994).

[12] S. Mrówczyński, Phys. Rev. D56, 2265 (1997).

[13] C. Greiner and S. Leupold, Ann. Phys. 270, 328 (1998).

[14] P.A. Henning, Nucl. Phys. A582, 633 (1995); Nucl. Phys. A586, 777 (1995); Phys. Rep. 253, 235 (1995).

[15] P. Bożek, Phys. Rev. C56, 1452 (1997).

[16] P. Bożek, in: M. Bonitz (ed.), Progress in Nonequilibrium Green's Functions, World Scientific, Singapore (2000).

[17] Yu.B. Ivanov, J. Knoll, and D.N. Voskresensky, Nucl. Phys. A657, 413 (1999); Nucl. Phys. A672, 313 (2000).

[18] M. Effenberger, E.L. Bratkovskaya, and U. Mosel, Phys. Rev. C60, 44614 (1999);

[19] M. Effenberger and U. Mosel, Phys. Rev. C60, 51901 (1999).

[20] W. Cassing and S. Juchem, Nucl. Phys. A665, 377 (2000).

[21] S. Leupold, Nucl. Phys. A672, 475 (2000).

[22] W. Cassing and S. Juchem, Nucl. Phys. A672, 417 (2000); Nucl. Phys. A677, 445 (2000).

[23] P. Danielewicz and S. Pratt, Phys. Rev. C53, 249 (1996).

[24] S. Leupold, in: M. Bonitz (ed.), Progress in Nonequilibrium Green's Functions, World Scientific, Singapore (2000).

[25] M.L. Goldberger and K.M. Watson, Collision Theory, Wiley, New York (1964).

[26] S.A. Bass et al., Prog. Part. Nucl. Phys. 41, 225 (1998).

[27] M.E. Peskin and D.V. Schroeder, An Introduction to Quantum Field Theory, AddisonWesley, New York (1995).

[28] J.M. Blatt and V.F. Weisskopf, Theoretical Nuclear Physics, Wiley, New York (1952). 


\section{FIGURES}

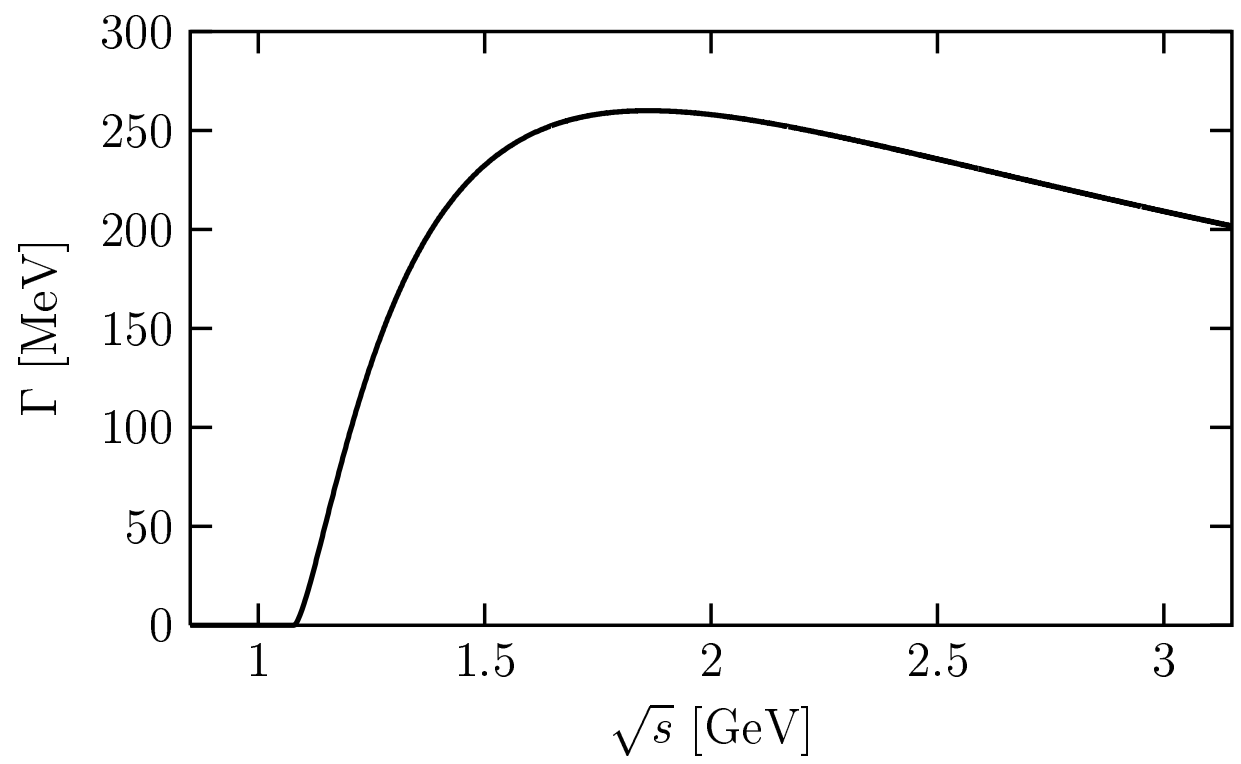

FIG. 1. Width $\Gamma$ as given in (A1) as a function of the invariant mass.

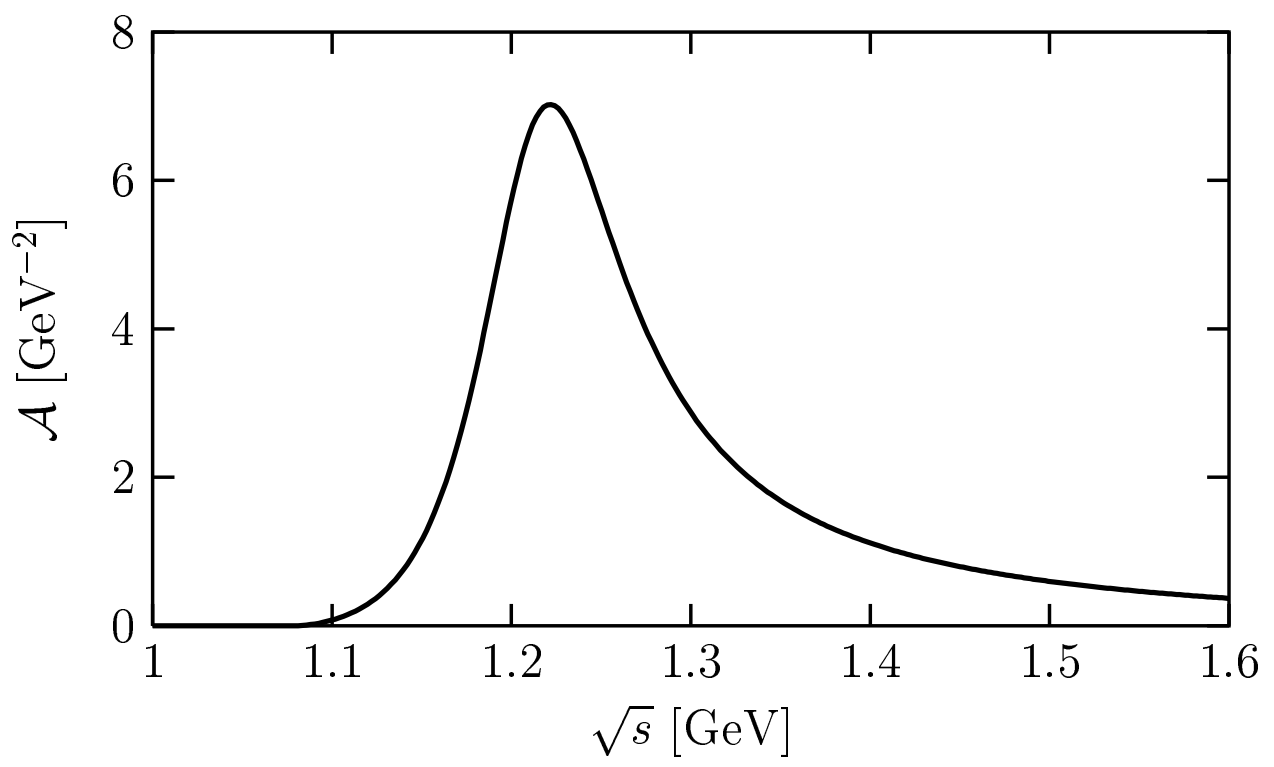

FIG. 2. Spectral function $\mathcal{A}$ as given in (2.10) as a function of the invariant mass. 


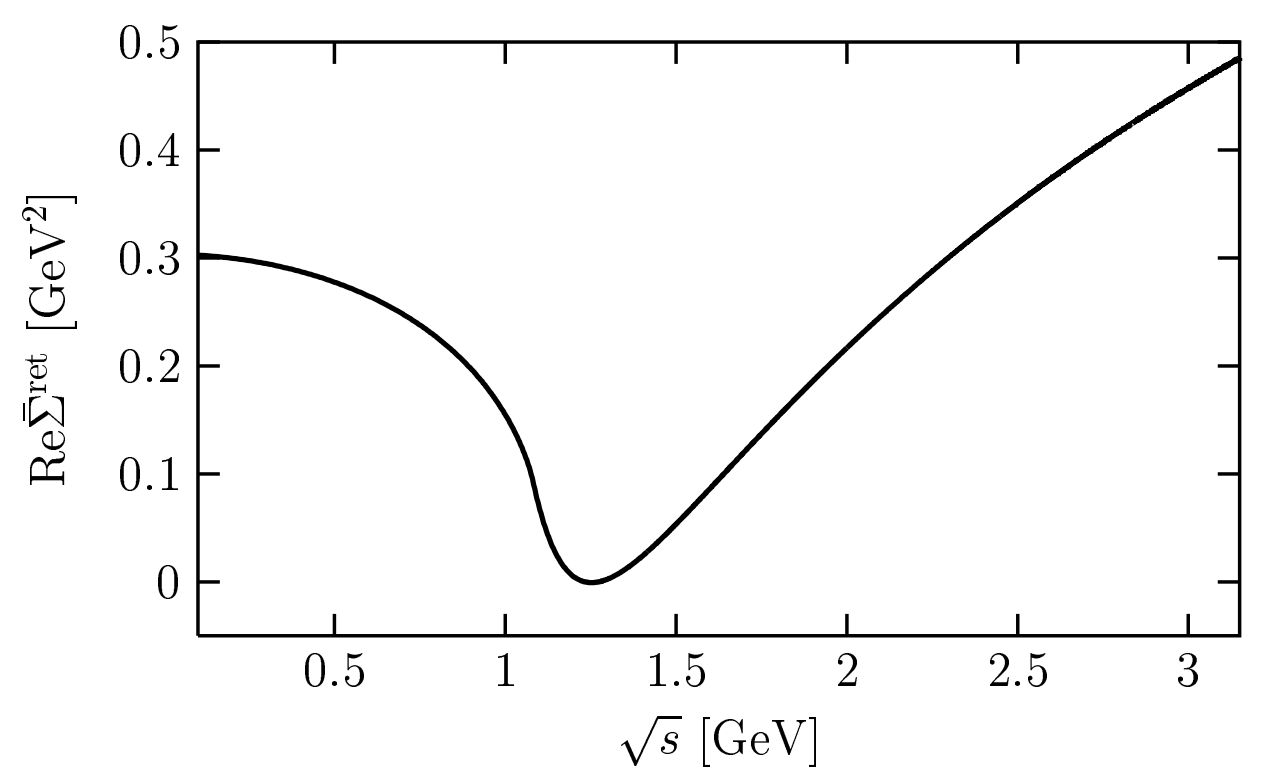

FIG. 3. Real part of the self-energy as given in (A4) as a function of the invariant mass.

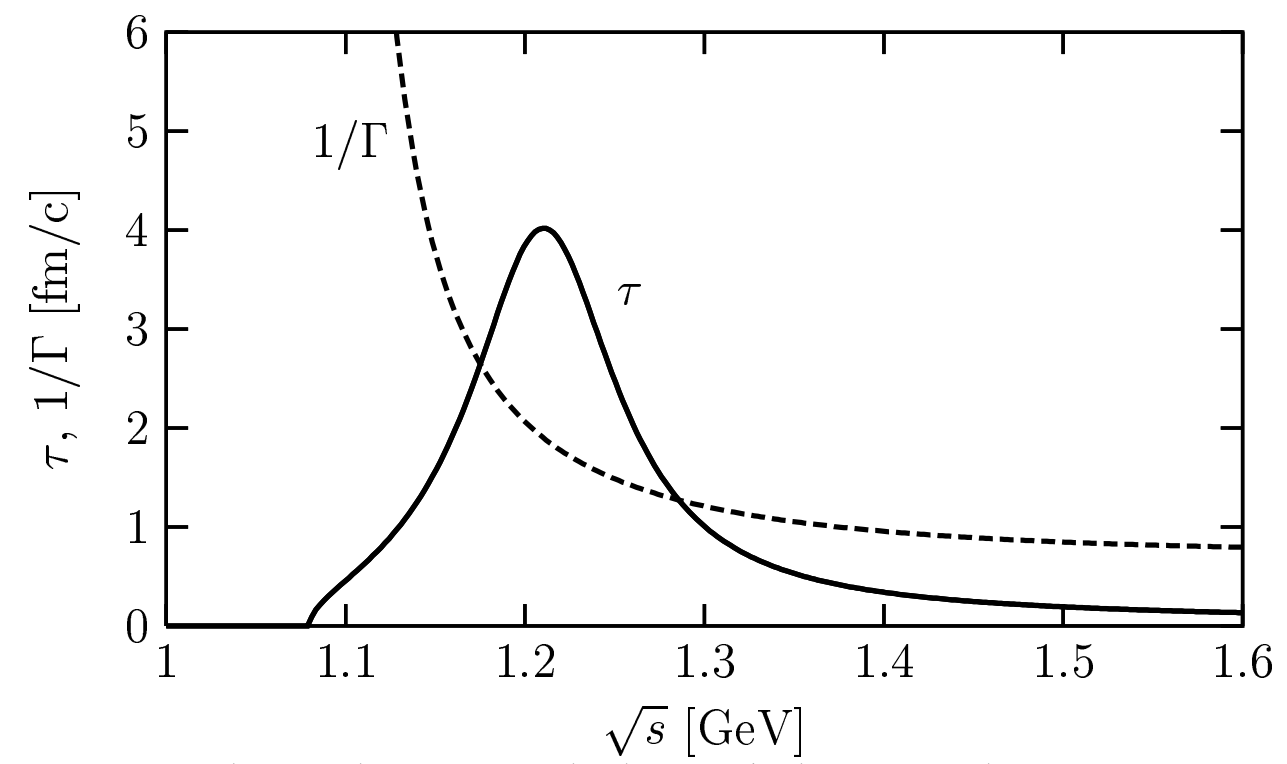

FIG. 4. Life time $\tau$ (full line) as given in (3.3) and $1 / \Gamma$ (dashed line) as functions of the invariant mass. Note that $\tau$ is evaluated in the rest frame of the decaying resonance, i.e. $p_{0}=\sqrt{s}$. 


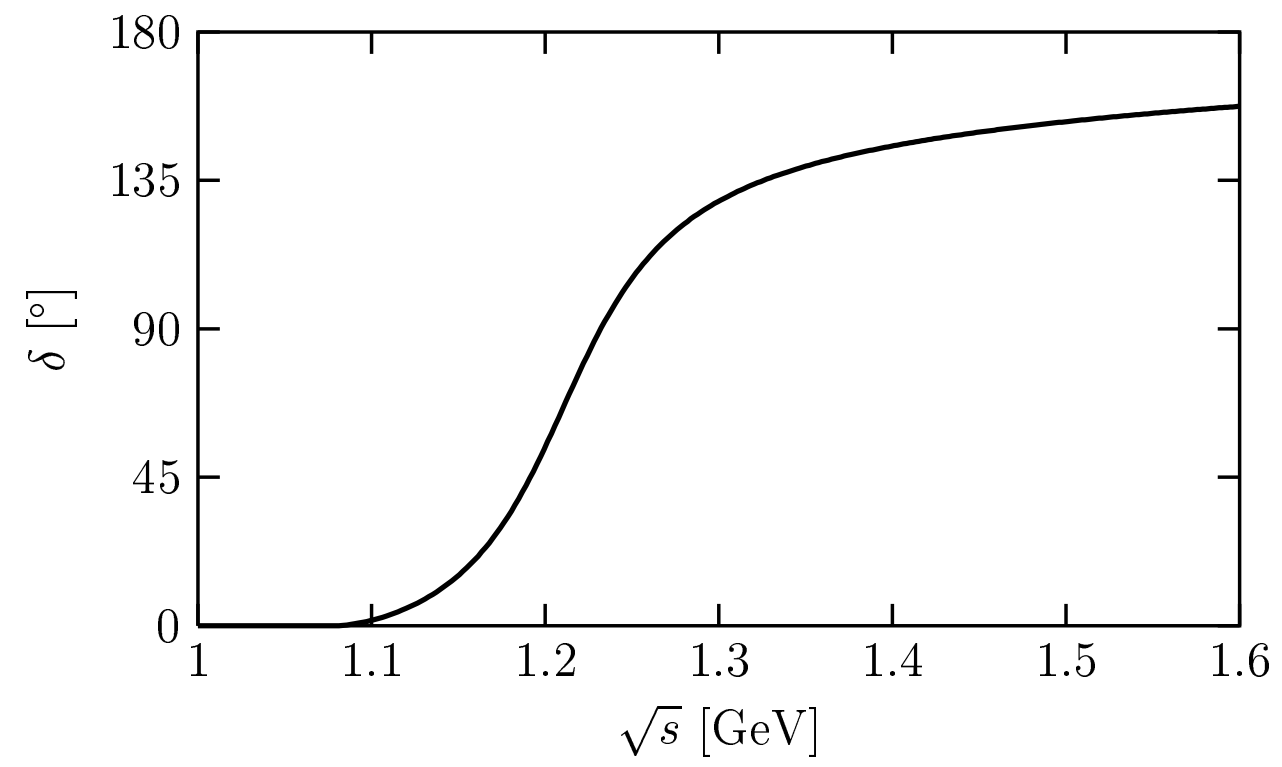

FIG. 5. Phase shift $\delta$ as defined in (3.4) as a function of the invariant mass.

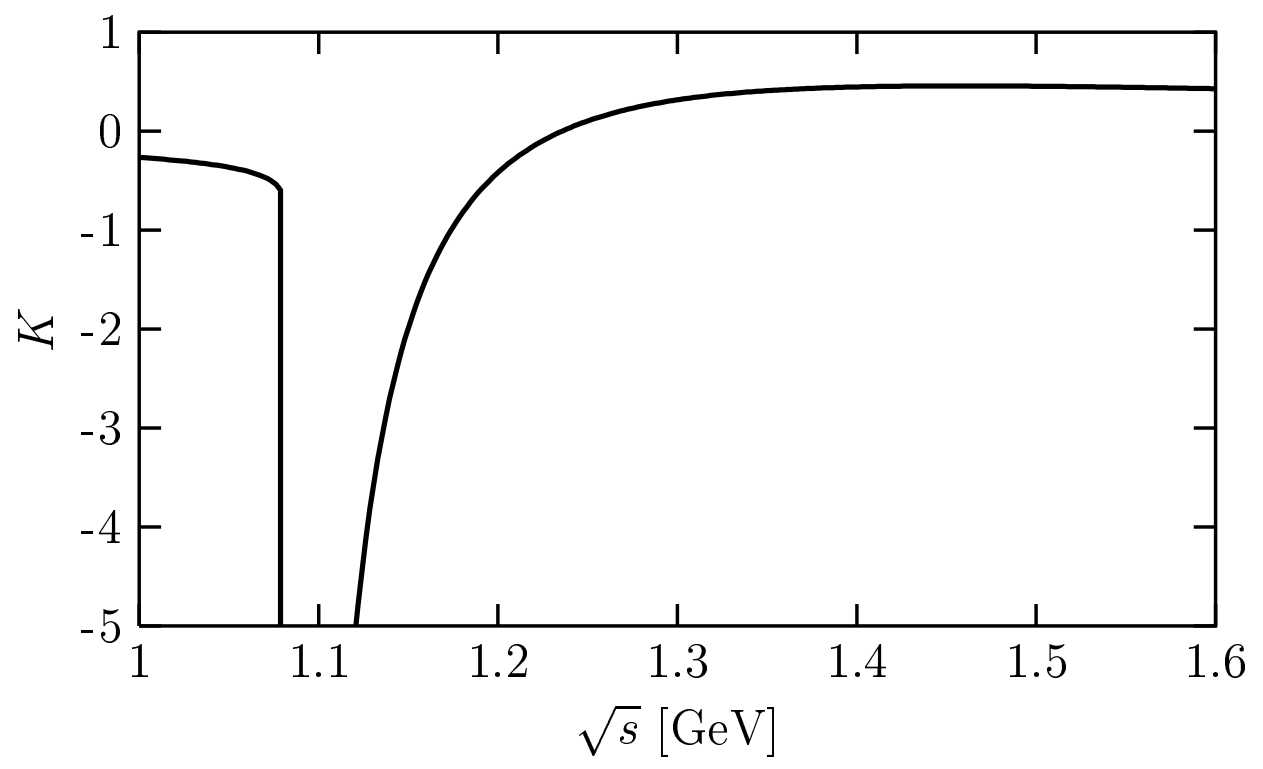

FIG. 6. $K$ as given in (2.23) as a function of the invariant mass. 


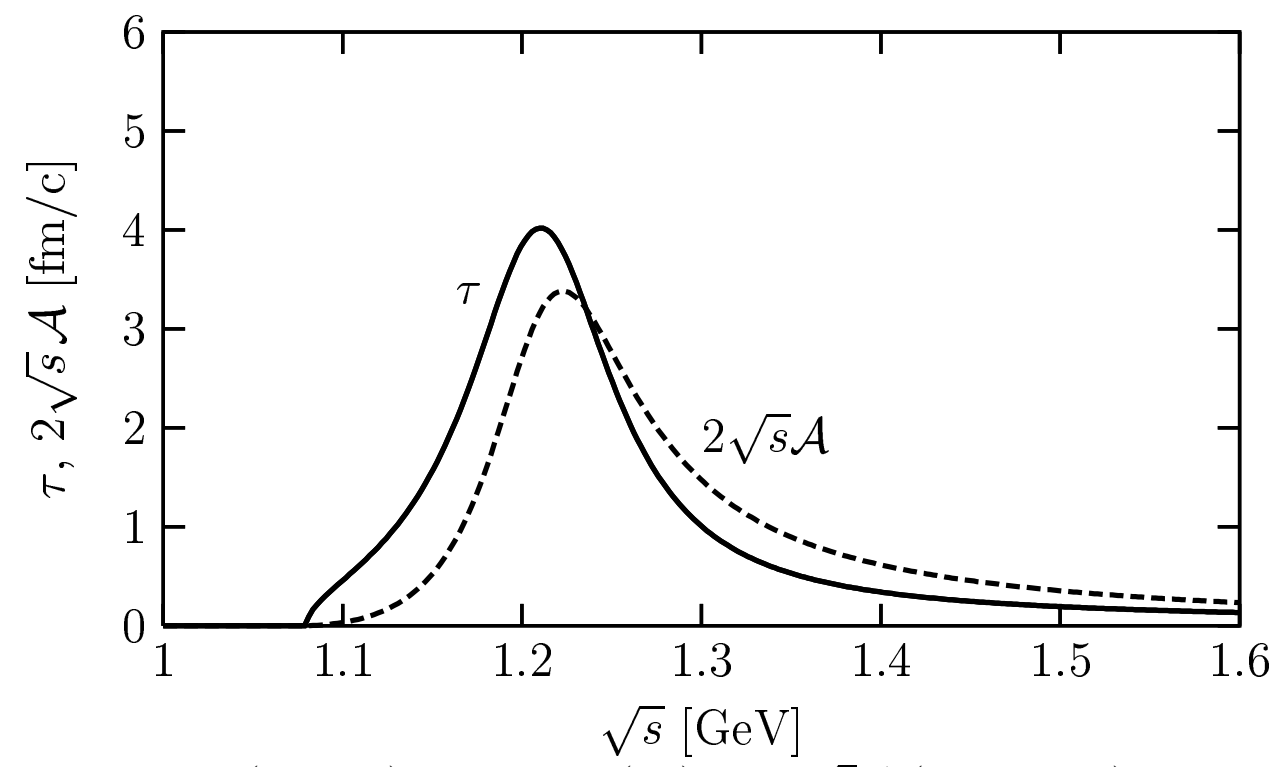

FIG. 7. Life time $\tau$ (full line) as given in $(3.3)$ and $2 \sqrt{s} \mathcal{A}$ (dashed line) as functions of the invariant mass. Note that $\tau$ is evaluated in the rest frame of the decaying resonance, i.e. $p_{0}=\sqrt{s}$. 